Published in final edited form as:

Cancer Epidemiol Biomarkers Prev. 2011 August ; 20(8): 1798-1801. doi:

10.1158/1055-9965.EPI-11-0441.

\title{
Circulating insulin-like growth factor-1 in pregnancy and maternal risk of breast cancer
}

\author{
Adetunji T Toriola ${ }^{1,2}$, Eva Lundin ${ }^{3}$, Helena Schock ${ }^{4}$, Kjell Grankvist ${ }^{3}$, Eero Pukkala ${ }^{5,6}$, \\ Tianhui Chen ${ }^{4}$, Anne Zeleniuch-Jacquotte ${ }^{7,8}$, Paolo Toniolo ${ }^{8,9,10}$, Matti Lehtinen ${ }^{6}$, Helja- \\ Marja Surcel ${ }^{1}$, and Annekatrin Lukanova ${ }^{4,9}$
}

${ }^{1}$ National Institute for Health and Welfare, Oulu, Finland ${ }^{2}$ Division of Preventive Oncology, National Center for Tumor Diseases, German Cancer Research Center, Heidelberg, Germany ${ }^{3}$ Department of Medical Biosciences, University of Umeå, Umeå, Sweden ${ }^{4}$ Division of Cancer Epidemiology, German Cancer Research Center, Heidelberg, Germany ${ }^{5}$ Finnish Cancer Registry, Institute for Statistical and Epidemiological Cancer Research, Helsinki, Finland ${ }^{6}$ Tampere School of Public Health, University of Tampere, Tampere, Finland ${ }^{7}$ New York University Cancer Institute, New York, USA ${ }^{8}$ Department of Environmental Medicine, New York University School of Medicine, New York, USA ${ }^{9}$ Department of Obstetrics and Gynecology, New York University School of Medicine, New York, USA ${ }^{10}$ Institute of Social and Preventive Medicine, Centre Hospitalier Universitaire Vaudois, University of Lausanne, Lausanne, Switzerland

\section{Abstract}

Background-Elevated serum concentrations of insulin-like growth factor (IGF)-1 have been associated with increased risk of breast cancer. Previously, we reported a similar association in samples obtained during pregnancy. The current study was conducted to further characterize the association of IGF-1 during pregnancy with maternal breast cancer risk.

Methods-A case-control study was nested within the Finnish Maternity Cohort. The study was limited to primiparous women less than 40 years of age, who donated blood samples during early (median, 12 weeks) pregnancy and delivered a single child at term. Seven hundred and nineteen women with invasive breast cancer were eligible. Two controls $(n=1,434)$ were matched to each case on age and date at blood donation. Serum IGF-1 concentration was measured using an Immulite 2000 analyzer. Conditional logistic regression was used to estimate odds ratios (OR) and 95\% confidence intervals (CI).

Results-No significant associations were observed between serum IGF-1 concentrations and breast cancer risk in both the overall analysis (OR 1.08 (95\% CI 0.80-1.47) and in analyses stratified by histological subtype, lag-time to cancer diagnosis, age at pregnancy or age at diagnosis.

Conclusion-There was no association between IGF-1 and maternal breast cancer risk during early pregnancy in this large nested case-control study.

Impact-Serum IGF-1 concentrations during early pregnancy may not be related to maternal risk of breast cancer.

\section{Keywords}

insulin-like growth factor-1 (IGF-1); pregnancy; breast cancer; nested case-control study

Correspondence and request for reprints: Annekatrin Lukanova, MD, PhD, Division of Cancer Epidemiology, German Cancer Research Center, In Neuenheimer Feld 581, Heidelberg 69120, Germany. Fax: +496221 422203; a.lukanova@dkfz.de. 


\section{Introduction}

Previously, in a study nested within the Northern Sweden Maternity Cohort (NSMC) we observed that insulin-like growth factor (IGF)-1 measured mostly during the first trimester of a primiparous pregnancy were positively associated with maternal risk of breast cancer [1], consistent with observations in non-pregnant women [2]. To confirm our initial findings and explore the association in greater detail we conducted a study with a very similar design, nested in the Finnish Maternity Cohort (FMC), the world's largest biorepository of serum samples from pregnant women.

\section{Materials and Methods}

\section{Selection of cases and controls}

Study design has been described in detail previously [3]. In brief, FMC members who donated serum samples between the $6^{\text {th }}$ and 14 th gestational weeks of a primiparous, singleton full-term pregnancy, younger than age 40 and with no history of in-situ breast or any other cancer (except non-melanoma of the skin) were eligible. Case subjects were 535 women with breast cancer identified through linkage with the Finnish Cancer Registry. As IGF-I concentration does not vary with gestational age during early pregnancy 184 cases with no data on exact gestational age were also included. For each case, two controls were matched on age at sampling ( \pm 6 months) and date of sampling ( \pm 3 months), for a total of 1,434 controls. Ninety two percent of the cases were less than 50 years at the time of diagnosis, thus the vast majority were likely diagnosed during fertile.

The study was approved by the ethical committee of the National Institute for Health and Welfare, Finland.

\section{Laboratory analyses}

IGF-1 assay was quantified by immunometric assays on the Immulite 2000 Siemens analyzer. The inter-run coefficients of variation $(\mathrm{CV})$ of the laboratory quality controls were $9.3 \%$ and $3.2 \%$ at concentrations of $81.5 \mathrm{ng} / \mathrm{mL}$ and $229 \mathrm{ng} / \mathrm{mL}$ respectively. The inter-run and intra-run CVs for a blinded pool of controls (mean concentration of $177 \mathrm{ng} / \mathrm{mL}$ ) were $3.9 \%$ and $8.3 \%$ respectively.

\section{Statistical analysis}

Prior to analysis, IGF-1 values were $\log 2$-transformed to normalize their distributions. The correlation of IGF-1 with gestational age was assessed by Pearson's partial correlation ( $\mathrm{r}=$ -0.03 ). Subjects were categorized into quintiles based on IGF-1 distribution among the controls. Conditional logistic regression was used to calculate the odds ratio and corresponding 95\% confidence interval (OR, 95\% CI) of breast cancer across quintiles of IGF-1. The associations were also explored by histological subtypes, median ages at first full term pregnancy ( 29 years) and diagnosis ( 41 years) and lag-time to cancer diagnosis (11 years) and also in finer subgroups of the latter 3 variables. Analyses limited to women with information on gestational age and by tertiles of time in storage were also conducted. Adjustment for potential confounders (gravidity, parity by index date, family history of breast cancer, smoking and gestational day) sporadically changed risk estimates but with less than 5\% and were not retained in the final model. Similarly, adjustment for estradiol (available for 534 case-control sets) had negligible effect on risk estimates. All statistical tests were two-sided and p-values $<0.05$ were considered statistically significant. 


\section{Results}

Selected characteristics of the study population and IGF-1 concentrations are presented in Table 1. Cases and controls were comparable in all characteristics except for family history of breast cancer.

There was no association of breast cancer with IGF-1 concentrations overall and in all the subgroup analyses (by histology, age at sampling, age at diagnosis, lag-time to diagnosis, storage time) (Table 2). Similarly, in analysis limited to case-control sets with information on gestational age $(n=535)$, there was no association of breast cancer with IGF-1 and adjustment for gestational age did not alter the risk estimates.

\section{Discussion}

In contrast to our previous findings in the NSMC (1), in the FMC, IGF-1 during early pregnancy was not associated with maternal risk of breast cancer. The two studies had very similar design and were nested in population-based maternity cohorts in neighboring countries. The samples are stored at comparative temperature $\left(-25^{\circ} \mathrm{C}\right)$ and the mean IGF-1 concentrations in the FMC controls $(134.7 \mathrm{ng} / \mathrm{mL})$ were comparable to those from the NSMC $(133.6 \mathrm{ng} / \mathrm{mL})$. IGF-1 was analyzed in the same laboratory with the same assay kits. The current study is 3 times larger and had $87 \%$ statistical power to detect an OR of 1.50 . Nevertheless, we cannot exclude the possibility that some analyte degradation has occurred and reduced our ability to find an existing association.

Another limitation of our study is the lack of information on estrogen-receptor (ER) status of the tumors, as this is not collected centrally in Finland. Most of the cases were diagnosed before age 50 (92\%) and thus more likely to be ER negative (4). The analysis by the Endogenous Hormones and Breast Cancer Collaborative Group suggested that the association of IGF-1 with breast cancer is confined to hormone-receptor positive tumors (2). Thus a relatively large proportion of receptor negative tumors in our data could have obscured an association with hormone-receptor positive disease.

In summary, no association between IGF-1 concentrations during early pregnancy and maternal breast cancer risk was observed in the FMC.

\section{Acknowledgments}

This work was supported by research grants from the US National Cancer Institute [CA114329 and CA120061]. The funding source had no role in the study design, interpretation of data and publication of results. Adetunji T Toriola was supported by a European Association for Cancer Research (EACR) Travel Fellowship Award to visit the Division of Cancer Epidemiology, German Cancer Research Center, Heidelberg. We appreciate the excellent technical assistance provided by Pirjo Kontiokari, Annika Uimonen, and Sara Kuusiniemi in the conduct of the study.

\section{References}

1. Chen T, Lukanova A, Grankvist K, Zeleniuch-Jacquotte A, Wulff M, Johansson R, Schock H, Lenner P, Hallmans G, Wadell G, Toniolo P, Lundin E. IGF-I during primiparous pregnancy and maternal risk of breast cancer. Breast Cancer Res Treat. 2010; 121(1):169-175. [PubMed: 19728079]

2. Key TJ, Appleby PN, Reeves GK, Roddam AW. Endogenous hormones and breast cancer collaborative group. Insulin-like growth factor 1 (IGF1), IGF binding protein 3 IGFBP3), and breast cancer risk: pooled individual data analysis of 17 prospective studies. Lancet Oncol. 2010; 11(6): 530-542. [PubMed: 20472501] 
3. Lukanova A, Surcel HM, Lundin E, Kaasila M, Lakso HA, Schock H, Husing A, Kaaks R, Koskela P, Grankvist K, Pukkala E, Zeleniuch-Jacquotte A, Lehtinen M, Toniolo P. Circulating estrogens and progesterone during primiparous pregnancies and risk of maternal breast cancer. Int $\mathrm{J}$ Cancer. 2011 (in press).

4. Dunnwald LK, Rossing MA, Li CI. Hormone receptor status, tumor characteristics, and prognosis: a prospective cohort of breast cancer patients. Breast Cancer Res. 2007; 9(1):R6. [PubMed: 17239243] 
Table 1

Selected characteristics of breast cancer cases and controls, median $\left(10^{\text {th }}, 90^{\text {th }}\right)$ or $\mathrm{n}$ (percentage) from the Finnish Maternity Cohort, 1983-2006.

\begin{tabular}{|c|c|c|c|}
\hline Characteristic & Cases (719) & Controls $(1,434)$ & p-Value $a$ \\
\hline Maternal age during index pregnancy (years) & $29.2(22.9-37.0)$ & $29.1(22.8-37.0)$ & \\
\hline \multicolumn{4}{|l|}{ Grouped Age } \\
\hline$<25$ years & $189(26 \%)$ & $372(26 \%)$ & \\
\hline $25-29$ years & $194(27 \%)$ & $396(28 \%)$ & \\
\hline $30-34$ years & $176(24 \%)$ & $358(25 \%)$ & \\
\hline$\geq 35$ years & $160(22 \%)$ & $308(21 \%)$ & \\
\hline Gestational age (days) $b$ & $73(57-89)$ & $73(57-90)$ & 0.48 \\
\hline Gravidity & $534(79 \%)$ & $1088(81 \%)$ & 0.33 \\
\hline Parity by index date & & & 0.10 \\
\hline 1 & $257(36 \%)$ & $463(32 \%)$ & \\
\hline 2 & $312(43 \%)$ & $652(45 \%)$ & \\
\hline$\geq 3$ & $150(21 \%)$ & $319(22 \%)$ & \\
\hline Age at diagnosis (years) & $40.9(32.1-49.3)$ & & \\
\hline Lag time (years) & $11.3(4.4-17.9)$ & & \\
\hline \multicolumn{4}{|l|}{ Histology } \\
\hline ductal carcinoma & $574(80 \%)$ & & \\
\hline lobular carcinoma & $98(14 \%)$ & & \\
\hline medullary carcinoma & $19(3 \%)$ & & \\
\hline other & $28(4 \%)$ & & \\
\hline Family history of breast cancer & $74(11 \%)$ & $62(5 \%)$ & $<0.0001$ \\
\hline Family history of ovarian cancer & $4(1 \%)$ & $13(1 \%)$ & 0.40 \\
\hline Smoking & & & 0.23 \\
\hline no & $443(84 \%)$ & $910(86 \%)$ & \\
\hline yes & $87(16 \%)$ & $149(14 \%)$ & \\
\hline Child sex & & & 0.74 \\
\hline male & $360(50 \%)$ & $729(51 \%)$ & \\
\hline female & $359(50 \%)$ & $705(49 \%)$ & \\
\hline Child birth weight $(\mathrm{g}){ }^{c}$ & $3,500(2,970-4,100)$ & $3,510(2,930-4,140)$ & 0.99 \\
\hline Child birth length $(\mathrm{cm}) d$ & $50(48-53)$ & $50(48-53)$ & 0.55 \\
\hline $\operatorname{IGF} \mathrm{I}(\mathrm{ng} / \mathrm{mL}) e^{e}$ & $133.7(94.9-198.0)$ & $134.7(94.5-195.0)$ & 0.53 \\
\hline \multicolumn{4}{|c|}{ Comparison between cases and controls: Conditional logistic regression models; } \\
\hline \multicolumn{4}{|c|}{${ }^{c}$ Data on child's birth weight is available for 537 cases and 1,077 controls } \\
\hline
\end{tabular}




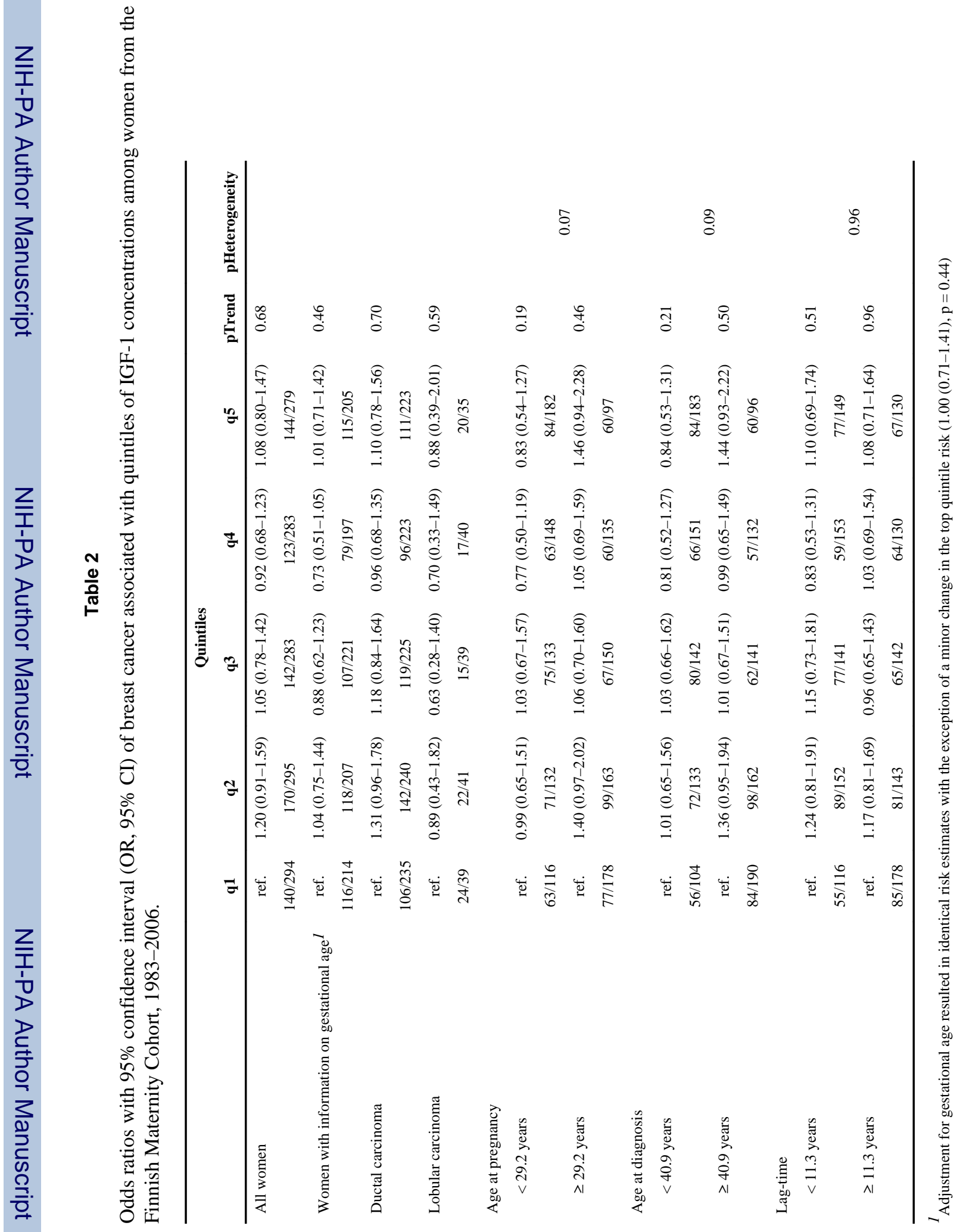

